\title{
Review of neonatal hypoglycaemia and adverse neurological outcomes
}

\author{
Dr Roisin Begley, ST5 Paediatrics \\ Dr Bushra Rafique, Speciality registrar \\ Dr Olajumoke Davies, Specialty doctor \\ Dr MAS Ahmed, Paediatric Consultant
}

Queens Hospital, Barking, Havering and Redbridge University Hospitals NHS Trust, Romford, Essex, UK

\section{Introduction}

Neonatal hypoglycaemia is a well-recognized cause of adverse neurological outcomes. While hypoglycaemic brain injury is well reported in the literature there is limited data on the effect of neonatal hypoglycaemia solely with other risk factors for brain injury excluded.

\section{Aims}

The aim of study was to evaluate the long-term neurological outcomes in patients with a history of symptomatic neonatal hypoglycaemia and no other risk factors of brain injury.

\section{Methodology}

- Retrospective cohort study examined the outcome among children with history of symptomatic neonatal hypoglycaemia.

- Babies born from January 2008 to January 2014 admitted to the neonatal intensive care unit at Queens Hospital, Romford, UK: a total 6411 new born infants were identified.

- $13 \%$ of neonatal admissions (850/6411) were recorded as having neonatal hypoglycaemia.

- Exclusion criteria (see table)

- 94/855 were identified with solely neonatal hypoglycaemia a risk factor for poor neurological outcome

- Data was reviewed from neonatal discharge summary, community assessments, ophthalmology, paediatric medical notes and laboratory results.

- 2/94 patients were found to have adverse neurological outcomes. These two cases are presented and analysed

\section{Results}

- 94 neonates with neonatal hypoglycaemia and no other risk factors for brain injury.

- 2/94 patients were found to have adverse neurological outcomes and neonatal seizures.

- The remaining 92/94 had favourable neurological outcome and no neonatal seizures.

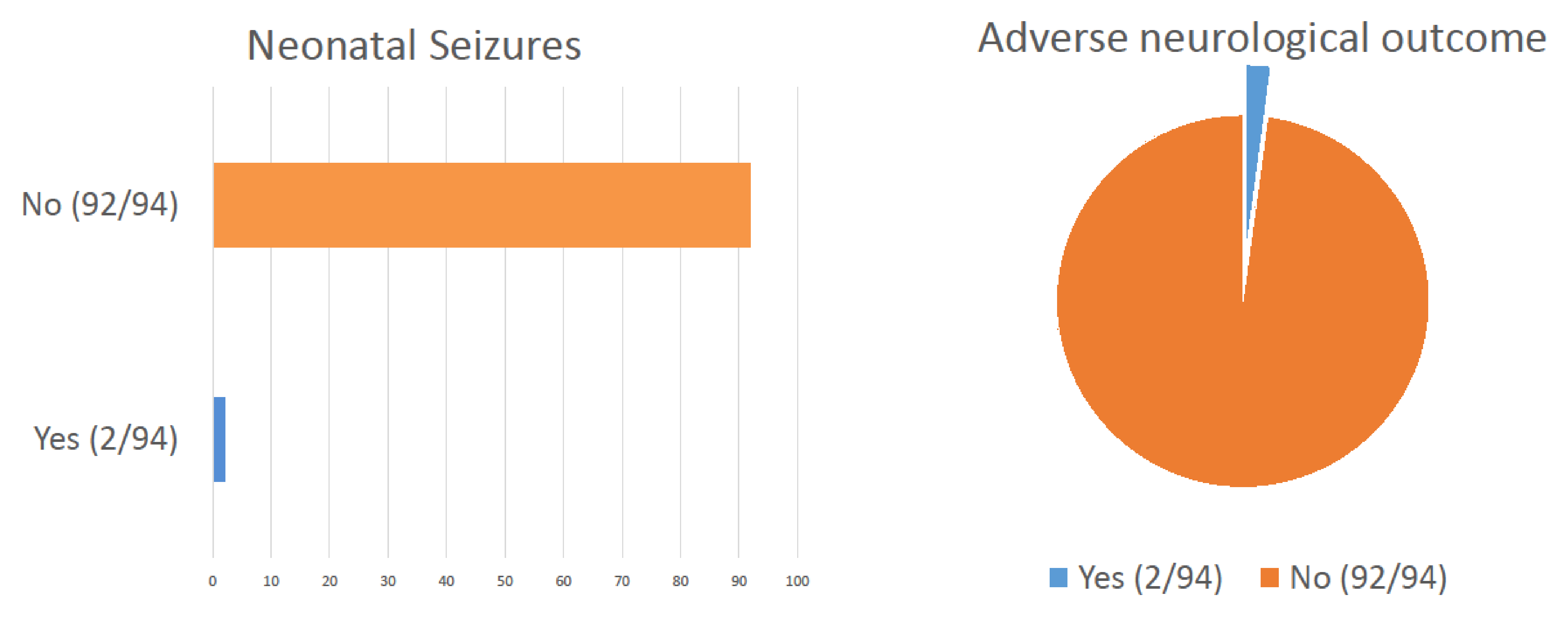

\section{Conclusions}

- Neonatal hypoglycaemia with no other risk factors for global brain injury can cause adverse neurological outcome.

- Neonatal seizure is a significant predictor of the potential long-term poor outcomes of isolated neonatal hypoglycaemia.

- Further research on the effect of neonatal hypoglycaemia and neonatal hypoglycaemic seizure on long-term neurological outcomes in needed.

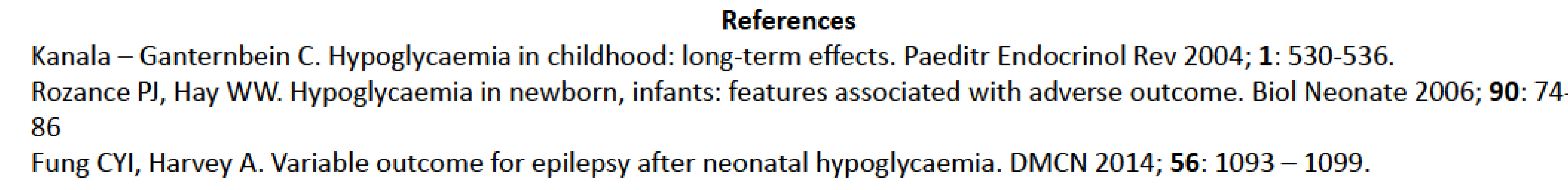

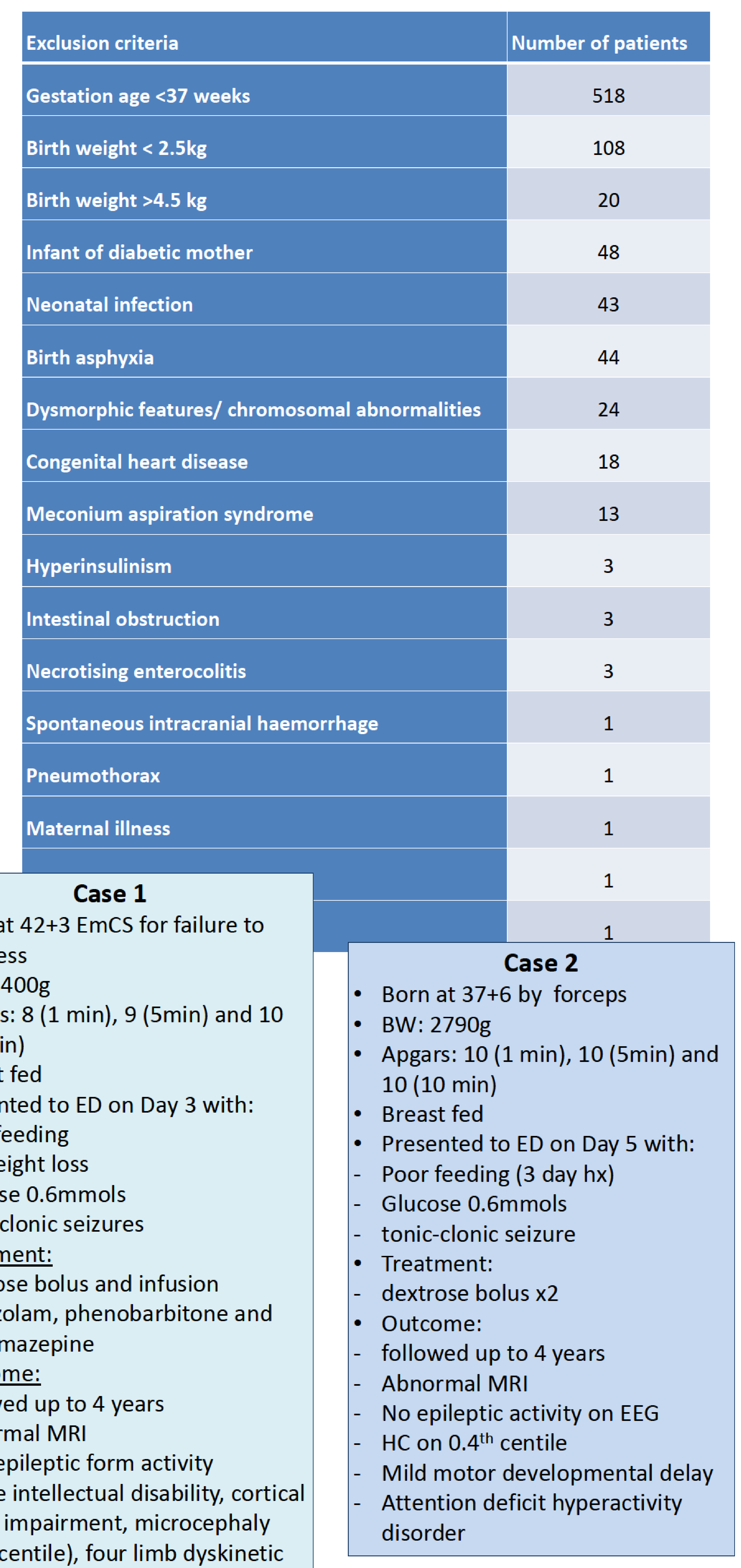

